\title{
SCFs study of tubular T/Y steel joints under inplane loading
}

\author{
Lalitesh Kumar' ${ }^{1}$, Ajay Kumar ${ }^{1, *}$, Danuta Barnat-Hunek ${ }^{2}$,Elżbieta Szczygielska ${ }^{3}$, and Monika Garbacz ${ }^{4}$ \\ ${ }^{1}$ National Institute of Technology, Department of Civil Engineering, 800005 Patna, India \\ ${ }^{2}$ Lublin University of Technology, Faculty of Civil Engineering and Architecture, Nadbystrzycka 40, 20-618 Lublin, Poland \\ ${ }^{3}$ Pope John Paul II State School of Higher Education in Biała Podlaska, Faculty of Economics and Engineering, Sidorska 95/97, \\ 21-500 Biała Podlaska, Poland \\ ${ }^{4}$ Lublin University of Technology, Faculty of Environmental Engineering, Nadbystrzycka 40B, 20-618 Lublin, Poland
}

\begin{abstract}
Stress concentration factors (SCFs) at welded tubular joints are one of the prime factors that affect the fatigue life of a structure. In the present work, finite element analysis (FEA) is used to find the hot spot stress and subsequently the stress concentration factors of Tubular T/Y steel Joints. Static axial tensile loading case is used in the present work. The circular hollow sections (CHS) are considered. The parametric study of the variation in SCF, with the change in joint angle $(\Theta)$ and geometrical parameters such as $\beta, \tau, \gamma$ for T/Y-Joints subjected to inplane axial loading, is done. The validation of FE modelling technique of present work is done by comparing with the various SCFs equations available in the literature
\end{abstract}

\section{Introduction}

Due to impressive technological advancement in the recent era, the requirement of a structure capable of being both strong as well as aesthetic is on the rise. Tubular members i.e. hollow steel sections fit the abovementioned criteria superbly. These tubular sections possess characteristic properties such as enhanced torsional rigidity, high weight to strength ratio, etc. Owing to these attributes, it is imperative for the tubular sections to reduce their construction cost and enhance the strength simultaneously.

Circular hollow sections (CHS) are the most widely used tubular sections. When two or more tubular members are welded together, the connection is known as tubular joint. It is obvious that there is a structural discontinuity at these connections. This causes concentration of stress at these joints, making these joint locations most susceptible to cracks and failure when subjected to fatigue loading. Fatigue is the phenomenon, which arises due to cyclic loading and it reduces the serviceable life of structure significantly. Therefore, the stress concentration factor (SCF) is a parameter, which can directly be used for the fatigue life estimation of these joints.

Extensive research studies have been conducted for the prediction of stress concentration factors ( $\mathrm{SCFs}$ ) at tubular joints in recent years. These authors aimed at studying several types of tubular joints for their behaviour under different loading conditions and boundary conditions. Kuang et al. [1] proposed empirical equations for stress concentration factors for simple nonreinforced T, K and KT-joints. They performed thin shell finite element analysis without considering any weld profile. In addition, only axial loading and inplane bending loads were considered. Moreover, Wordsworth and Smedley [2] rectified these shortcomings by considering out of plane bending and a new type of joint called X-joints. They formulated equations based upon the acrylic model test results. However, their equations were only valid for the weld crown and saddle locations. They ignored the intermediate weld sections. UEG [3] recommended a modification factor in Wordsworth and Smedley [2] equations for higher $\beta$ and $\gamma$. Efthymiou and Durkin [4] again analysed welded tubular T-joint by FEA (Finite Element Analysis) using a shell element for the calculation of SCF. They considered the weld bead effect and incorporated it in their results. Kuang et al. [1], Gibstein [5], Romeijn [6], Karamanos [7] and Hellier [8] studied a welded tubular T-joint by using the Marc Finite element program. Since there are numerous FEA software, Pang and Lee [9] and Chang and Dover [10], both opted for the finite element program ABAQUS. Both used thin-shell elements with five degrees of freedom per node in order to study an X-joint. N'Diaye [11] studied the SCF of welded, notched tubular T-joint under axial, bending and dynamic loading. Yong-Bo [12] emphasised on the stress distribution of $\mathrm{T}$ and $\mathrm{K}$-joint under axial loading. Thibaux and Cooreman [13] calculated the SCF for Yjoint using solid quadratic element. They studied the SCF for both outer and inner portion of the tube under different loading conditions. Saini et al. [14] presented a brief review of the SCF of the tubular joint. They reviewed all the important SCF equations and different section such as circular as well as square. Apart from these studies, there are various design guidelines such as

\footnotetext{
${ }^{*}$ Corresponding author: sajaydce@gmail.com
} 
American Petroleum Institute (API) [15] and CIDECT Design Guide No. 8 [16], which recommends the use of hot spot stress methods for SCF estimation.

These authors focused their studies on static loading. Despite all these efforts, SCF in tubular joints is still a debatable topic and a more detailed study is required. Therefore, an effort has been made in this research work to study maximum SCF in brace members of tubular $\mathrm{T} / \mathrm{Y}$-joints by using FEA package ABAQUS. In this research work, the hot spot stress from the FEA is used to calculate the SCF in tubular T-joints with different non-dimensional parameters. The research includes the SCF variation with respect to different non-dimensional parameters such as $\tau, \beta$, and $\gamma$. In addition, a validation study is performed for the FE model generation technique, in order to check the authenticity of the technique used.

\section{$2 \mathrm{~T} / \mathrm{Y}$-joint geometry and various non- dimensional parameters}

Since the present research work covers the effect of the variation of different parameters such as $\tau, \beta$ and $\gamma$ on $\mathrm{SCF}$, the dimensions of studied tubular $\mathrm{T} / \mathrm{Y}$ joints have been varied accordingly. The procedure for altering the dimensions to change the non-dimensional parameters is explained in further paragraphs. The non-dimensional parameters are presented below. Fig. 1 shows a typical $\mathrm{T} / \mathrm{Y}$-tubular joint.

Wall thickness ratio, $\tau=\frac{t}{T}$

Diameter ratio, $\quad \beta=\frac{d}{D}$

Chord stiffness, $\quad \gamma=\frac{D}{2 T}$

Chord length parameter $\alpha=\frac{2 L}{D}$

where $\mathrm{L}=$ length of the chord,

$\mathrm{D}=$ chord outside diameter

$\mathrm{T}=$ chord wall thickness,

$\mathrm{d}=$ brace outside diameter,

$\mathrm{t}=$ brace wall thickness, $\Theta=$ brace angle

Notably, the literature survey suggested that these parameters have certain validity ranges. Therefore, for this research work, the validity range of CIDECT Design Guide No. 8 [14] is selected. These parameters are varied in their specified ranges and SCF is calculated in these ranges. These ranges are cited below. The symbols have their usual meaning as discussed above.

$$
\begin{gathered}
0.2<\tau<1.0 \\
0.2<\beta<1.0 \\
8<\gamma<32 \\
30^{\circ}<\Theta<90^{\circ}
\end{gathered}
$$

The FE models are created using DS Catia and ABAQUS. For non-dimensional parameters variations, dimensions of models are manually selected to obtain the desired non-dimensional parameter. The scheme of varying dimensions of FE models for each nondimensional parameter are explained in detail below.

\subsection{Dimensions of the FE models for $\tau$-variation}

For $\mathrm{t}$-variation, only " $\mathrm{t}$ " is varied while keeping the other dimensions constant. The constant dimensions are $\mathrm{L}=$ $1000 \mathrm{~mm}, \mathrm{D}=200 \mathrm{~mm}, \mathrm{~d}=100 \mathrm{~mm}, \mathrm{~T}=10 \mathrm{~mm}$. " $\mathrm{t}$ " is varied as $2 \mathrm{~mm}, 4 \mathrm{~mm}, 6 \mathrm{~mm}, 8 \mathrm{~mm}$ and $10 \mathrm{~mm}$. A separate model is developed for each " $t$ ". The length of the brace is half to that of the length of the chord (brace length is measured from the centreline of the chord) i.e. 1 $=500 \mathrm{~mm}$. The FE models were created at $\Theta=90^{\circ}, 60^{\circ}$, $30^{\circ}$.

\subsection{Dimensions of the FE models for $\boldsymbol{\beta}$-variation}

For $\beta$-variation, only $d$ is varied, while keeping other dimensions is constant. The dimensions that remain constant are $\mathrm{L}=1000 \mathrm{~mm}, \mathrm{D}=200 \mathrm{~mm}, \mathrm{~T}=10 \mathrm{~mm}, \mathrm{t}=$ $6 \mathrm{~mm}$. "d" is varied as $40 \mathrm{~mm}, 80 \mathrm{~mm}, 120 \mathrm{~mm}$ and 160 $\mathrm{mm}$. Similar method was followed to develop a separate model for each "d". The length of the brace was same as that of in $\tau$-variation i.e. $500 \mathrm{~mm}$ (from the centreline of chord). The FE models are created for different brace angles $\Theta=90^{\circ}, 60^{\circ}, 30^{\circ}$.

\subsection{Dimensions of the models for $\boldsymbol{\gamma}$-variation}

For $\gamma$-variation, only $\mathrm{T}$ is varied, and all the other dimensions are kept constant. The constant dimensions are $\mathrm{L}=1000 \mathrm{~mm}, \mathrm{D}=200 \mathrm{~mm}, \mathrm{~d}=100 \mathrm{~mm}$ and $\mathrm{t}=2 \mathrm{~mm}$. The dimension "T" is varied as $10 \mathrm{~mm}, 5 \mathrm{~mm}$ and 3.33 $\mathrm{mm}$. The brace angle variation is done same as the above. However, the change in the value of " $T$ " results in the change in $\tau$ and $\gamma$ simultaneously. This factor is considered while modelling and result discussion.

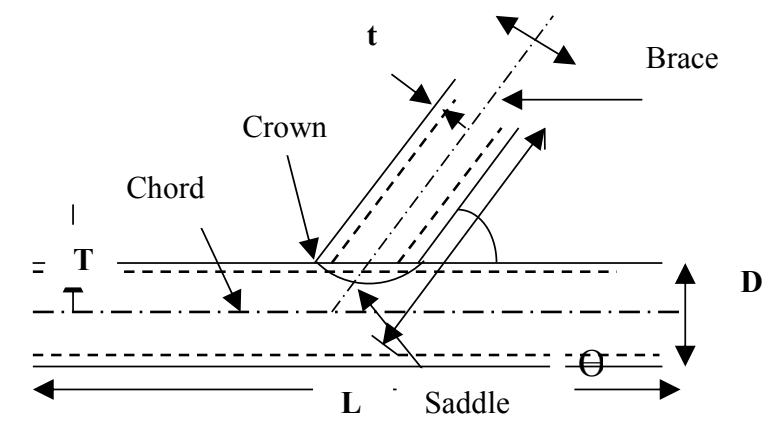

Fig. 1 A typical T/Y tubular joint.

\section{Finite element modelling}

The precision of FE modelling of tubular joints directly affects the SCF values. Hence, the FE-model generation procedure is opted quite similar to the previous authors. In present work, the modelling procedure involves two software packages. In the first stage, the chord and brace are modelled separately in the assembly module of the DS Catia. The elements selected for both brace and chord are 3D solid elements. In the second stage, these separate components i.e. brace, and chord are imported to ABAQUS and merged together in the assembly module of ABAQUS, thus generating a tubular joint. The fillet weld profile is modelled using the chamfer tool in ABAQUS. The weld size is taken constant for all the models i.e. $5 \mathrm{~mm}$ fillet length size. Fig. 2 and 3 show the FE modelling of the tubular $\mathrm{T}$ and $\mathrm{Y}$-joint along with the fillet weld, respectively.

Since tubular sections are generally made up of steel, properties of steel are used in the ABAQUS.

1) Young Modulus (E): $210000 \mathrm{MPa}$ 
2) Density ( $\rho): 0.0000078 \mathrm{~kg} / \mathrm{mm}^{3}$

3) Poisson's Ratio (v): 0.30

4) Weld length $=5 \mathrm{~mm}$ (for all the FE models)

The SCF values are very specific values as altering the boundary conditions, changes it accordingly. For present research work, both the chord ends of the joint is kept fixed in case of all the models. The fixed boundary condition is $\mathrm{U} 1=0, \mathrm{U} 2=0, \mathrm{U} 3=0, \mathrm{UR} 1=0, \mathrm{UR} 2=0$, $\mathrm{UR} 3=0$. This boundary condition restricts the displacements and rotations in all directions. The SCF value in this research work is assumed for the fixed boundary conditions.

A uniformly distributed load of $1 \mathrm{MPa}$ tensile is applied at the brace end surface in all the FE models. Applying this load makes the nominal stress in brace member equal to $1 \mathrm{MPa}$.

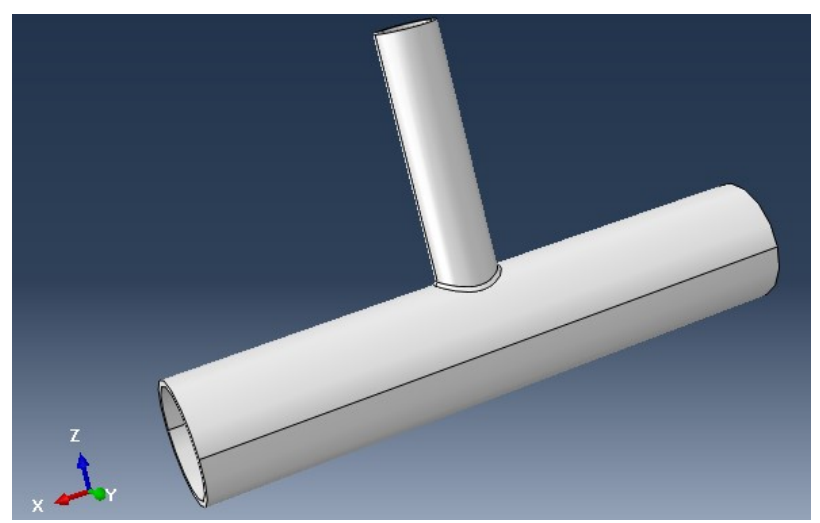

Fig. 2. T-joint model in ABAQUS.

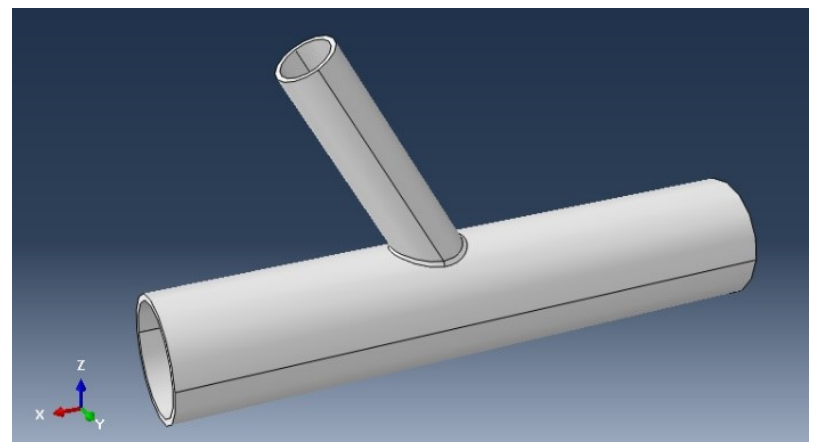

Fig. 3. Y-joint $\left(60^{\circ}\right)$ model in ABAQUS.

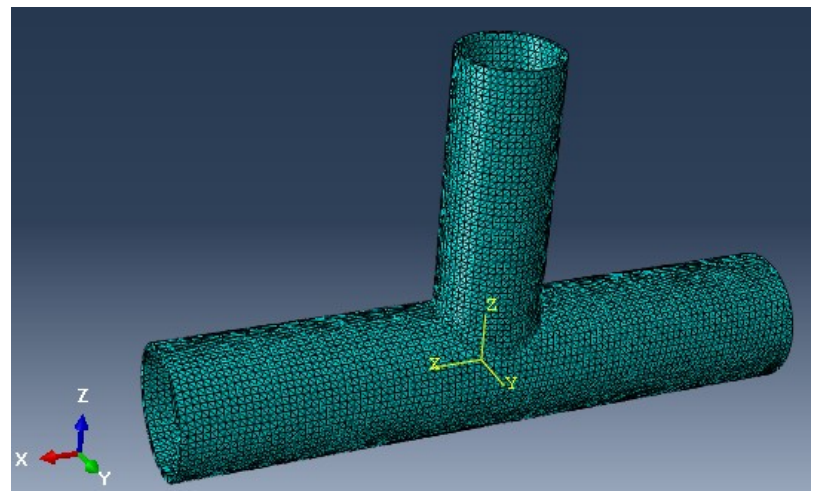

Fig. 4. Meshed T-joint in ABAQUS.

For FEA, ABAQUS element type C3D10 has been used to model tubular joint components i.e. the chord, brace and the weld profiles. C3D10 are continuum (solid) second order tetrahedral elements. This type of element has 10 nodes with six degrees of freedom and 4 integration points. This element type foster zero nodal force at the corner nodes upon the application of pressure load on a face. C3D10 elements are recommended for any contact involving finite sliding with node to surface or surface-to-surface formulation when used with the penalty enforcement method. The penalty enforcement method is the default method in ABAQUS for contact enforcement. Due to these factors, C3D10 are used for better results. In the present work, since the mesh size selected itself is very fine, no mesh refinement at the weld profile is considered. Fig. 4. shows the meshed tubular T-joint. A global mesh size of 10 is selected to mesh all the FE models.

\section{Stress concentration factor (SCF)}

SCFs can be defined as the ratio between hot spot stresses developed at the welded joint to the nominal stresses.

$$
S C F=\frac{\sigma_{\text {hot spotstress }}}{\sigma_{\text {nominal }}}
$$

The FE analysis provides the hot spot stress values i.e. maximum stress values. For simplicity, the brace summit is loaded with a uniformly distributed load of 1 MPa tensile load. Hence, the nominal stress is equal to the $1 \mathrm{MPa}$ and the hot spot stress is equal to the SCF. Also, from the literature, it is evident that hot spots are observed at the weld section in the tubular joint and this hot spot stress can directly be read from the FEA. Some authors select Von Mises stress from FEA while others prefer principle stresses for fatigue analysis. However, Von Mises stress are a better parameter for the analysis. Hence, for all the FEA analysis in the present work, the hot spot stress is read from Von Mises stress value from the FEA results. The SCF study has been done for the brace member only.

\section{Results and discussions}

The pre-discussed procedure is opted to model all the $\mathrm{T} / \mathrm{Y}$-joints. In order to check the accuracy and reliability of the models, the same procedure is opted to model the tubular joints of N'Diaye [11] and the calculated SCF value is compared with the author's one. Once the procedure is validated, the same procedure is followed to model other tubular joints with different geometrical parameters. Thereafter, SCF is calculated using ABAQUS based upon the hot spot stress analysis. A parametric study is done for maximum SCF in brace member with respect to different non-dimensional parameters $(\tau, \beta$, and $\gamma)$. A detailed discussion is carried out regarding the obtained results.

\subsection{Validation study}

For validation study, N'Diaye [11] tubular T-joint model is selected. It is a CHS-CHS section joint. The dimensions are taken from [11] and are modelled according to the present modelling techniques. The same 
3D-solid element as all the other models i.e. C3D10 is used to model the selected T-joint. The mesh is done with the option of mesh by instance with a global size of 20. The fillet weld size is selected as $5 \mathrm{~mm}$ length by using chamfer tool with no rounding cut. The maximum SCF calculated in the brace is obtained at the brace saddle position. Table 1 tabulates the dimensions of $\mathrm{T}$ joint [11] used for validation. Table 2 introduces the material properties assigned to the $\mathrm{T}$-joint.

Table 1. Dimensions of the tubular T-joint [11].

\begin{tabular}{|c|c|}
\hline $\mathrm{L}=4130 \mathrm{~mm}$ & $1=2065 \mathrm{~mm}$ \\
\hline $\mathrm{D}=508 \mathrm{~mm}$ & $\mathrm{~d}=406 \mathrm{~mm}$ \\
\hline $\mathrm{T}=12.7 \mathrm{~mm}$ & $\beta=0.5 \mathrm{~mm}$ \\
\hline$\tau=0.75$ & $\alpha=16.25$ \\
\hline$\gamma=20$ & $\mathrm{t}$ \\
\hline \multicolumn{2}{|c|}{ Length of the fillet weld $=5 \mathrm{~mm}$} \\
\hline
\end{tabular}

Table 2. Properties of the welded T-joint for validation.

\begin{tabular}{|c|c|}
\hline E, Young's modulus & $207 \mathrm{GPa}$ \\
\hline$\nu$, Poisson's ratio & 0.3 \\
\hline$\rho$, Material density & $0.000007800 \mathrm{Kg} / \mathrm{mm}^{3}$ \\
\hline
\end{tabular}

Table 3. Comparison of the SCF calculated in brace member with the results available in literature.

\begin{tabular}{|c|c|}
\hline $\begin{array}{c}\text { Inplane Axial tensile } \\
\text { loading }\end{array}$ & Loading case \\
\hline 8.6 & UEG [3 ] \\
\hline 9.6 & Kuang et al. [1] \\
\hline 11.2 & Gibstein [5] \\
\hline 12.8 & Efthymiou and Durkin [4] \\
\hline 11.7 & Hellier et al.[8] \\
\hline 9.62 & N'diaye [11] \\
\hline 10.73 & Present work \\
\hline
\end{tabular}

Table 3 unveils the comparison results of the SCF obtained by the [11] and different standard SCF equations to the current evaluated SCF. It shows that the maximum SCF value obtained for the brace member is 10.73 and it is located at the saddle portion of the brace. The table also compares the present result with the other author's results. The SCF calculated matches best with the values obtained by Gibstein [5]. The SCF calculated herein is for the inplane static axial loading. It is also observed that there is slight variation in SCF with some authors and it could be due to different modelling technique. In addition, some authors use principle stresses while some use Von Mises stress as hot spot stress. Based on table 3, it can be said that the opted procedures for the modelling as well as FEA analysis are in good agreement with the other authors and the same procedure can be used for finding out new results at different inputs. The different inputs are the various nondimensional parameters $\tau, \beta$, and $\gamma$. The variation of maximum SCF is computed only for the saddle portion of the brace member. The detailed variation of SCF is stated in the following subsections with the help of graphs and tables.

\subsection{Effect of $\tau$-variation on SCFs}

$\tau$ represents the ratio of the brace thickness to the chord thickness. As the $\tau$ increases, the brace thickness increases correspondingly. Fig 5 displays the variation of maximum SCF in brace member with the nondimensional parameter $\tau$ at different brace angles: $90^{\circ}$, $60^{\circ}$ and $30^{\circ}$. The graph is plotted with constant nondimensional parameters, $\alpha=10, \beta=0.5, \gamma=10$. Upon investigating the graph below, it is observed that SCF increases along with the increase in $\tau$ value for each $\Theta$. The increment slope is much higher in the case of higher $\Theta$. It is worth notable that for maximum SCF in the case of $90^{\circ}$ and $60^{\circ}$, the location of maximum SCF is saddle portion of brace. However, the location of maximum SCF shifts towards the brace crown portion in case of $30^{\circ}$ brace. This can be generalized for lesser $\Theta$ i.e. $\Theta \approx$ $30^{\circ}$, maximum $\mathrm{SCF}$ is at crown locations. The $\mathrm{SCF}$ results for $30^{\circ}$ tubular joint in fig. 5 is actually the SCF at the crown locations of brace. The SCF at $90^{\circ}$ brace is almost three times to that of $30^{\circ}$ brace for $\tau=1$ i.e. both chord and brace thickness are same. For a single joint, for instance, $90^{\circ}$ brace, the SCF increases almost 4 times from $\tau=0.2$ to $\tau=1.0$. Moreover, for a single joint, e.g. $\Theta=$ constant, the value of SCF increases following a non-linear curve. The increment slope is greater for the higher inclination joints. The variation of SCF shows the same trend for all $\Theta$, i.e. a strictly increasing curve. This variation may be attributed to the fact that with the increase in $\tau$, enhanced brace thickness impart more structural discontinuity at the intersection and the stress increases at that locations. Apart from this, the weld volume increases with increase in $\tau$, this could also be one of the reasons for this pattern of variation.

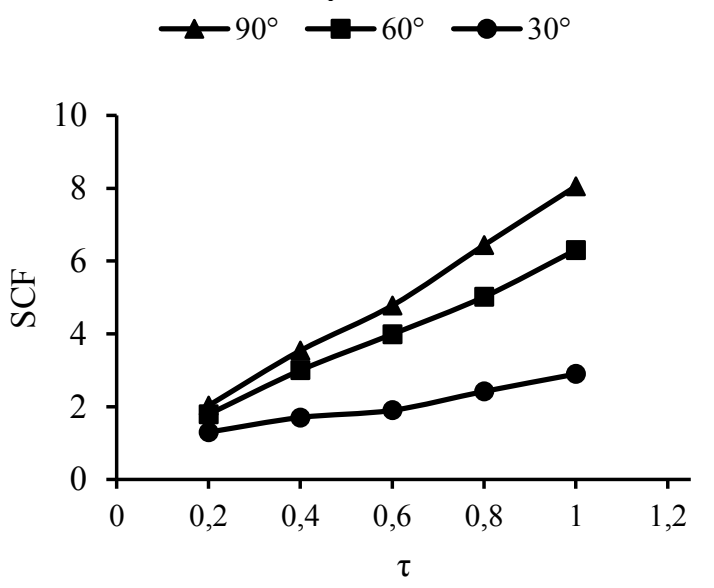

Fig. 5. Variation of SCF vs $\tau$ for T/Y-joint for $\alpha=10, \beta=0.5$, $\gamma=10$ for different brace angle, CHS section. 


\subsection{Effect of $\beta$-variation on SCFs}

$\beta$, a non-dimensional parameter defined by the ratio of the brace diameter (outer) to the chord diameter (outer). As the $\beta$ value increases, the brace diameter grows correspondingly. However, there is a limitation for brace diameter as it can only be increased up to D. Fig 6 displays the variation of maximum SCF in brace with the non-dimensional parameter $\beta$ for different brace angle $90^{\circ}, 60^{\circ}$ and $30^{\circ}$. The graph is plotted with constant nondimensional parameters $\alpha=10, \tau=0.6, \gamma=10$. While FEA, it is noted that maximum SCF in brace member occur at the brace saddle for $90^{\circ}$ and $60^{\circ}$ while for the $30^{\circ}$ joint, the brace crown portion is the location of maximum SCF. Fig. 6 explains that SCF in the case of $90^{\circ}$ joint is maximum and the SCF value decreases for a further decrease in $\Theta$.

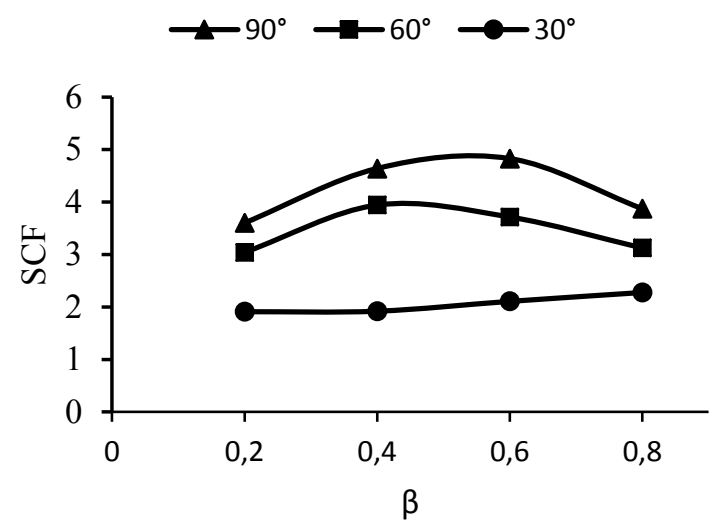

Fig. 6. Variation of SCF vs $\beta$ for T/Y-joint for $\alpha=10, \tau=0.6$, $\gamma=10$ for different brace angle, CHS section.

Table 4. Variation of maximum SCF in brace member of T/Yjoint with respect to $\gamma$ and $\tau(\alpha=10, \beta=0.5)$.

\begin{tabular}{|c|c|c|c|}
\hline T-Joint types & $\gamma$ & $\mathrm{T}$ & $\mathrm{SCF}$ \\
\hline \multirow{3}{*}{$90^{\circ} \mathrm{T}$-joint } & 10 & 0.20 & 2.03 \\
\cline { 2 - 4 } & 20 & 0.40 & 4.65 \\
\cline { 2 - 4 } & 30 & 0.60 & 8.75 \\
\hline \multirow{3}{*}{$60^{\circ}$ T-joint } & 10 & 0.20 & 1.79 \\
\cline { 2 - 4 } & 20 & 0.40 & 3.84 \\
\hline \multirow{3}{*}{$30^{\circ}$ T-joint } & 30 & 0.60 & 7.93 \\
\cline { 2 - 4 } & 10 & 0.20 & 1.30 \\
\cline { 2 - 4 } & 30 & 0.40 & 2.34 \\
\hline
\end{tabular}

The curve shows that the SCF value first increases and then decreases after attaining a maximum value close to $\beta=0.5$ for $90^{\circ}$ and $60^{\circ}$. However, in the case of $30^{\circ}$ joint, the maximum SCF is at the crown of brace and shows rising pattern along with an increase in $\beta$. In addition, the variation is not that pronounced as in the case of $\tau$ - variation. Again, for higher $\Theta$, higher SCF values are obtained.

\subsection{Effect of $\gamma$-variation on SCFs}

$\gamma$ represents the ratio of the diameter of chord to the twice of the thickness of the chord wall. The method followed to vary $\gamma$ has been discussed already in section 2.3. It can be observed that when the thickness of chord $\mathrm{T}$ is varied, $\tau$ is changed automatically. Table 4 shows the maximum SCF obtained in the brace member. Similar to the above-mentioned $\tau$ and $\beta$ variation, the maximum SCF is also obtained at brace saddle in $90^{\circ}$ and $60^{\circ}$, while the location of maximum SCF shifts at the brace crown in the case of $30^{\circ}$. The values of maximum SCF increase sharply along with the value of $\gamma$. One thing to note is that wall thickness of chord is reduced to increase $\gamma$. Hence, the decreased thickness of the chord can be the reason of the increased SCF values. It is obvious that the chord strength and stiffness is reduced significantly if the thickness is decreased and this reduced stiffness accounts for this steep rise in SCF. There is approximately a 4-fold rise in the SCF value when $\gamma$ changes from 10 to 30 in the case of all brace angles.

\section{Conclusion}

In view of the results presented above, following conclusions can be drawn.

a) Whenever a tubular joint is subjected to static axial loading, there is a stress concentration at the joint intersection.

b) For $\tau$ variation, maximum SCF in brace along increases with $\tau$ and maximum SCF is obtained when the brace thickness is equal to the chord thickness i.e. $\tau=1$. SCF vs $\tau$ is a strictly increasing curve for all $\Theta$. For $\Theta=30^{\circ}$, maximum SCF is obtained at brace crown portion while for $90^{\circ}$ and $60^{\circ}$, it is obtained at brace saddle portion.

c) For $\beta$ variation, maximum SCF in brace is obtained at brace saddle portion for $90^{\circ}$ and $60^{\circ}$. However, for $\Theta=30^{\circ}$, maximum SCF is obtained at the brace crown portion. It is a curve that increases first, attains a maximum value and then decreases. However, for $\Theta=30^{\circ}$, it is an ever-increasing curve.

d) For $\gamma$ variation, maximum SCF in the brace member increases along with the $\gamma$ value for all $\Theta$.

e) SCF in brace member for higher $\Theta$ is always greater than that for the lower $\Theta$.

\section{References}

1. J.G. Kuang, A.B. Potvin, R.D. Leick, Proceedings of the 7th annual offshore technology conference (OTC 2205, Houston TX, 593-612 1975) 
2. A.C. Wordsworth, G.P. Smedley, Proceedings of the European Offshore Steel Research Seminar, IX/P31, 1-7 (1978)

3. Underwater Engineering Group, Design Guidance on Tubular Joints in Steel Offshore Structures, (Report UR33, April, UK, 1985).

4. M. Efthymiou, S. Durkin, Proceedings of the Conf. on Behavior of Offshore structures (Elsevier, Amsterdam, Netherlands, 429-440, 1985).

5. M.B. Gibstein, Proceedings of the European offshore steel research seminar (Cambridge, UK, paper 26, 1978)

6. Romeijn A., Stress and strain concentration factors of welded multiplanar tubular-joints, $\mathrm{PhD}$ thesis. Delft University Press, 11 (1994)

7. S. Karamanos, A. Romeijn, J. Wardenier, Eng. Struct. 22 (1), 4-14 (2000)

8. A.K. Hellier, M.P. Connolly, W.D. Dover, Int. J. Fatigue 12(1), 12-23 (1990)

9. H.L.J. Pang, C.W. Lee, Int. J. Fatigue 17(5), 313$320(1995)$
10. E. Chang, W.D. Dover, Int. J. Fatigue 21(4), 361381 (1999)

11. A. N'Diaye, S. Hariri, G. Pluvinage, Z. Azari, Int. J. Fatigue 31, 367-374 (2009)

12. S. Yong-Bo, J. Constr. Steel Res. 63(10), 1351$1360(2007)$

13. P. Thibaux, S. Cooreman, Proceedings of the ASME 32nd International Conference on Ocean, Offshore and Arctic Engineering OMAE (2013)

14. D. S. Saini, D. Karmakar, S. Ray-Chaudhuri, J. Ocean Eng. Sci. 1(3), 186-202 (2016)

15. American Petroleum Institute (API), API RP 2AWSD, Recommended Practice for Planning, Designing and Constructing Fixed Offshore (Platforms: Working Stress Design, American Petroleum Institute, Washington, D.C, 2005)

16. X.L. Zhao, S. Herion, J.A. Packer, R. Puthli, G. Sedlacek, J. Wardenier, K. Weynand, A. Van Wingerde, N. Yeomans, Design Guide for Circular and Rectangular Hollow Section Joints under fatigue loading (CIDECT Publication, No. 8. Germany, TUV-Verlag, 2000). 